\title{
ELECTRICAL AND OPTICAL PROPERTIES OF HIGHLY NON-STOICHIOMETRIC GaAs*
}

\author{
A. Kurpiewski, K. Korona, M. KamińsKa \\ Institute of Experimental Physics, Warsaw University \\ Hoża 69, 00-681 Warszawa, Poland
}

M. PALCZEWSKA

Institute of Electronic Materials Technology, Wólczyńska 133, 01-919 Warszawa, Poland

\section{JAGADish AND J. Williams}

Department of Electronic Materials and Engineering, Australian National University

Canberra, Australia

The studies of transport and optical properties of GaAs implanted with high arsenic doses were performed. As-implanted samples showed hopping conductivity and the exponential absorption tail in the near-IR region. Both effects were probably caused by the amorphization of implanted layer. Using EPR measurements it was found that arsenic antisite defect with high local strain field was created during implantation. Annealing of implanted layers at $600^{\circ} \mathrm{C}$ led to substantial removal of amorphization, decrease in absorption coefficient and hopping conductivity leading to resistive samples. The possible model of such behaviour may be similar to the one of suggested for low temperature $\mathrm{GaAs}$ layers.

PACS numbers: $73.90 .+\mathrm{f}, 78.30 . \mathrm{Er}$

One of the recent attractive semiconductor material for electronics and optoelectronics is GaAs grown by molecular beam epitaxy at lower than normal substrate temperatures, about $200^{\circ} \mathrm{C}$. Grown at such low temperatures $\mathrm{GaAs}$ crystals (low temperature GaAs - LT GaAs) contain large amount of excess arsenic up to $1.5 \%$, which is found in antisite and non-regular interstitial positions [1,2]. Subjected to annealing at $600^{\circ} \mathrm{C}$ LT GaAs eliminates backgating when used as buffer layer in metal-semiconductor field-effect transistors and shortens response time in photoconductive switches and photodetectors down to few tens of picoseconds [3-5]. Although the mechanism of high resistivity and fast recombination of photocarriers in LT GaAs annealed at $600^{\circ} \mathrm{C}$ has not found a definite explanation

*This work is supported in part by the State Committee for Scientific Research (Republic of Poland) grant No. 3P 40702506. 
yet, the considered models appreciate the importance of high excess of arsenic in this non-stoichiometric material. The still discussed question is whether arsenic antisite defects or arsenic precipitates emerging during annealing are important for the properties of annealed LT GaAs. The aim of the presented paper was to create non-stoichiometric GaAs using implantation with high doses of arsenic ions and compare its properties with those of LT GaAs. The behaviour of implanted GaAs under annealing at $600^{\circ} \mathrm{C}$ was of special interest.

Semi-insulating GaAs wafers were used as a starting material for implantation with arsenic ions. The implantation with doses between $10^{13}$ to $10^{16}$ As ions $/ \mathrm{cm}^{2}$ was performed using Tandem Ion Implanter at the Australian National University. Annealing of implanted GaAs pieces was done under arsenic overpressure in a chamber of metallo-organic chemical vapour deposition system. Annealing time was 10 minutes. Additionally, the pieces of the sample implanted with $10^{16} \mathrm{As}$ ions $/ \mathrm{cm}^{2}$ were annealed for 1 and 2 hours.

Electron paramagnetic resonance (EPR) experiments were performed using Bruker ESP-300 X-band spectrometer. A helium gas-flow Oxford Instruments cryostat was used for variable temperature operation from $4 \mathrm{~K}$ up to $300 \mathrm{~K}$. Illumination of samples was performed with a 150 watt tungsten-halogen lamp. Absorption measurements were performed using Varian Cary 5 double beam spectrophotometer. The samples were mounted in the Air Products closed cycle helium refrigerator allowing the measurements at temperature range of 10 to $300 \mathrm{~K}$. Resistivity measurements were performed using a computerised setup with a picoammeter allowing for conductivity measurements with passing currents down to $1 \mathrm{pA}$.

The EPR quadruplet ascribed previously to $\mathrm{As}_{\mathrm{Ga}}^{+}$defect [6] was observed in GaAs samples implanted with doses of $10^{14} \mathrm{As}$ ions $/ \mathrm{cm}^{2}$ and higher. The characteristic feature of the signal was the lack of saturation in the temperatures below $10 \mathrm{~K}$, similarly to what has been reported for n-irradiated GaAs [7] and in opposite to observation in as-grown bulk material for which EPR signal has maximum at about $10 \mathrm{~K}$. Moreover, no EPR signal was observed from control substrate sample of the same dimensions. This means that the implanted layer was the only source of the EPR signal in the experiment. The amplitude of the As $\mathrm{s}_{\mathrm{Ga}}$ quadruplet increased with the implantation dose. In all the samples it was not sensitive for white light illumination. Annealing at $600^{\circ} \mathrm{C}$ led to decrease in EPR signal amplitude. It was observable only for samples implanted with doses greater than $5 \times 10^{15}$ As ions $/ \mathrm{cm}^{2}$ and was about three times smaller than before annealing.

Absorption spectra of all implanted samples (doses ranging from $10^{13}$ to $10^{16} \mathrm{As}$ ions $/ \mathrm{cm}^{2}$ ) showed an exponential tail in near-infrared (near-IR) region starting from photon energy of about $0.85 \mathrm{eV}$ and extending up to GaAs absorption edge (Fig. 1). The absorbance values measured between 0.85 and $1.5 \mathrm{eV}$ increased with the increase in implantation dose. About $2 \%$ of the observed absorption band could be quenched after white light illumination. Shape of the quenched absorption corresponded to the characteristic absorption of main GaAs defect, EL2 (arsenic antisite in neutral charge state). Using control non-implanted sample it was checked that the EL2 absorption comes from the starting semi-insulating (SI) $\mathrm{GaAs}$ crystal. The remaining dominant part of the absorption spectrum originated 

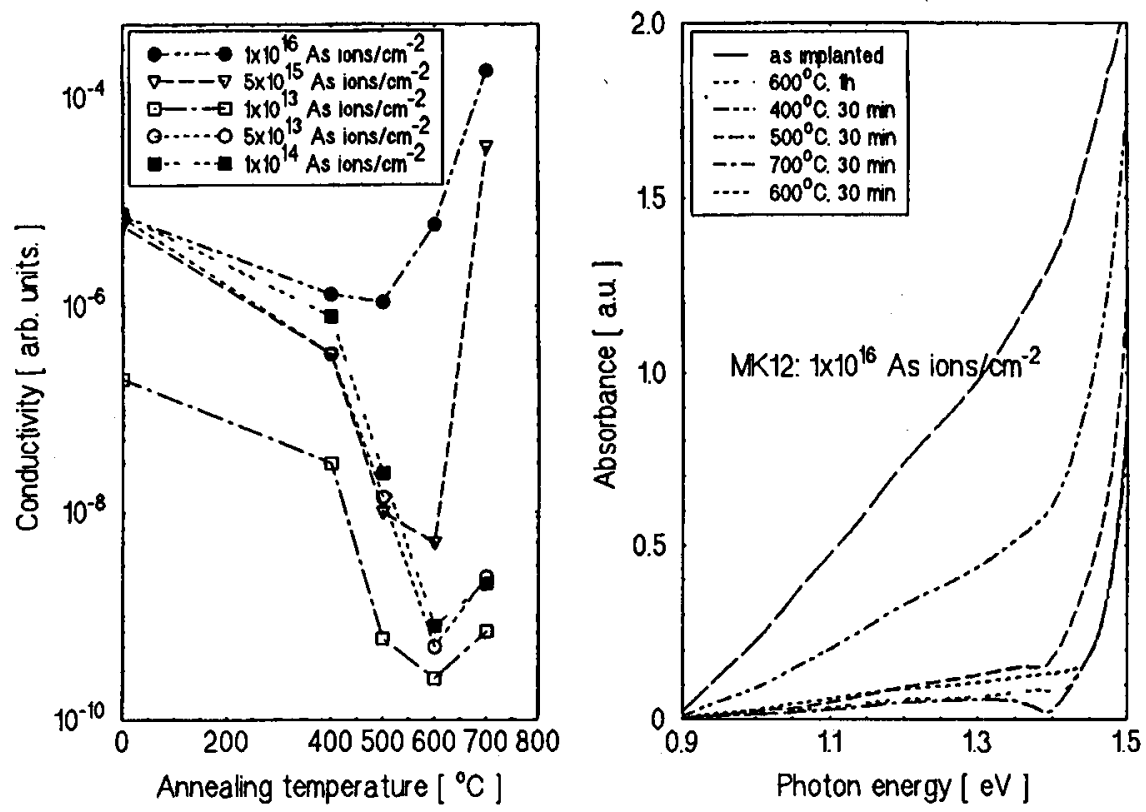

Fig. 1. Absorption spectra of the GaAs samples implanted with arsenic annealed at different temperatures. Implantation dose: $10^{16}$ As ions $/ \mathrm{cm}^{-2}$ (right) and conductivity of arsenic implanted GaAs samples as a function of annealing temperature (left).

from the implanted layer and was not quenchable with light. Starting from $400^{\circ} \mathrm{C}$, annealing of the implanted samples led to the gradual decrease in the exponential near-IR absorption tail.

Electrical transport measurements showed the decrease in resistivity of as implanted samples with increase in the implantation dose. The shape of temperature dependence of conductivity is characteristic of hopping type: for temperatures above $150 \mathrm{~K}$ the conductivity is described by equation: $\sigma=\sigma_{03} \exp \left(-\varepsilon_{3} / k T\right)(T-$ temperature). The hopping activation energy $\varepsilon_{3}$ is about $0.08 \mathrm{eV}$ for all measured samples. Below $140 \mathrm{~K}$ Mott's law: $\sigma=\sigma_{04} \exp \left[-\left(T / T_{0}\right)^{1 / 4}\right]$ is fulfilled. Annealing of implanted samples led to decrease in conductivity (Fig. 1). All implanted samples but the one implanted with the highest dose of $10^{16} \mathrm{As}$ ions $/ \mathrm{cm}^{2}$ showed the minimum of conductivity when annealed at $600^{\circ} \mathrm{C}$. The sample implanted with $10^{16} \mathrm{As}$ ions $/ \mathrm{cm}^{2}$ dose had slight minimum for annealing temperature of $500^{\circ} \mathrm{C}$, its conductivity decreased by less than order of magnitude, and then started to increase again for higher annealing temperatures. The conductivity of this sample annealed at $700^{\circ} \mathrm{C}$ was more than an order of magnitude higher than for as-implanted one. Samples implanted with doses between $5 \times 10^{13}-10^{14}$ As ions $/ \mathrm{cm}^{2}$ showed the highest decrease in conductivity (by more than three orders of magnitude) when annealed at $600^{\circ} \mathrm{C}$ in comparison with the conductivity of as-implanted sample. The conductivity of the samples implanted with doses lower than $5 \times 10^{13} \mathrm{As}$ ions $/ \mathrm{cm}^{2}$ changes less with annealing temperatures in comparison with the samples implanted with higher doses. 
The excess arsenic incorporated in GaAs crystals during implantation is partially found in form of arsenic antisite defect as seen from EPR measurements. The EPR signal of arsenic antisite defect created during implantation had features similar to the one of arsenic antisite in defected crystals: n-irradiated [7] or plastically deformed. The lack of saturation and quenchability at low temperatures has been previously connected with high local strain field of $\mathrm{As}_{\mathrm{Ga}}$ defect [8]. The strain field resulted most probably from damage created during implantation $[9,10]$. It has been determined from the independent channelling studies that for implantation doses used in the experiment, amorphization of GaAs implanted layer takes place indeed. The observed exponential tail of the near-IR absorption spectrum and hopping conductivity is also due to the amorphization. Annealing of the implanted layers led to the gradual recrystallization of the amorphised layer and therefore, the exponential tail together with the hopping conductivity decreased with annealing. Arsenic antisite defects created during implantation partially annealed out at $600^{\circ} \mathrm{C}$ and simultaneously the resistivity of implanted layers increased. Similar behaviour was observed for LT GaAs layers. Arsenic antisite defect present in those layers in concentrations up to $10^{20} \mathrm{~cm}^{-3}$ was found to anneal down to concentration about $10^{18} \mathrm{~cm}^{-3}$ at temperature of $600^{\circ} \mathrm{C}$. It was shown that arsenic precipitates are created during such annealing and arsenic antisite is most probably accommodated within them. On the other hand, it was found that LT GaAs annealed at $600^{\circ} \mathrm{C}$ increased its resistivity in comparison with as-grown layer which had hopping conductivity [11]. This high resistivity was linked with either the presence of arsenic precipitates or - according to competitive models - with arsenic antisite defects of still high concentration - about $10^{18} \mathrm{~cm}^{-3}$. It is too early to judge which of the above mechanisms is decisive in the case of annealed implanted layers but the similarity of behaviour of annealed implanted layers to LT GaAs behaviour is not questionable. EPR studies of implanted layers show that neutral charge state of arsenic antisite defect is not populated. Also no absorption due to the neutral charge state was observed. Therefore, the implantation process must lead to creation of acceptor defects besides of arsenic antisite defects and concentrations of both of them must be similar. It is difficult to say anything about the nature of the created acceptor defects since no EPR signal besides the one due to arsenic antisite was observed.

\section{References}

[1] K.M. Yu, M. Kamińska, Z. Liliental-Weber, J. Appl. Phys. 72, 2850 (1992).

[2] M. Kamińska, Z. Liliental-Weber, E.R. Weber, T. George, J.B. Kortright, F.W. Smith, B.Y. Tsaur, A.R. Calawa, Appl. Phys. Lett. 54, 1881 (1989).

[3] F.W. Smith, A.R. Calawa, C.-L. Chen, M.J. Manfra, L.J. Mahoney, IEEE Electron Device Lett. 9, 77 (1988).

[4] M.Y. Frankel, J.F. Whitaker, G.A. Mourou, F.W.Smith, A.R. Calawa, IEEE Trans. Electron Devices 37, 2493 (1990).

[5] Y. Chen, S. Williamson, T. Brock, F.W. Smith, A.R. Calawa, Appl. Phys. Lett. 59, 1984 (1991).

[6] E.E. Wagner, D.E. Mars, G. Hom, G.B.Stringfellow, J. Appl. Phys. 51, 5434 (1980). 
[7] J. Wosik, M. Palczewska, M. Kamińska, Acta Phys. Pol. A 69, 431 (1986).

[8] M. Hoikis, E.R. Weber, Phys. Rev. B 39, 3872 (1989).

[9] A. Kozanecki, B.J. Sealy, R. Gwilliam, P. Kidd, Nucl. Instrum. Methods Phys. Res. $B$ 80/81, 798 (1993).

[10] M.V. Rao, Nucl. Instrum. Methods Phys. Res. B 79, 645 (1993).

[11] K.P. Korona, M. Kamińska, J.M. Baranowski, E.R. Weber, Mater. Sci. Eng. B 22, 41 (1993). 